\title{
Determining the Forces in the Polyethylene Pipes after Squeezing them off with Specific Equipment
}

\author{
Avrigean Eugen $^{1,}$, Oleksik Mihaela Emilia ${ }^{2, b}$ \\ ${ }^{1}$ Lucian Blaga University, 10 Victoriei Blvd, 550024, Sibiu, Romania \\ ${ }^{2}$ Lucian Blaga University, 10 Victoriei Blvd, 550024, Sibiu, Romania \\ aeugen.avrigean@ulbsibiu.ro, ${ }^{\mathrm{b}}$ mihaela.oleksik@yahoo.com
}

Keywords: polyethylene pipe, external force, finite elements, simulation.

Abstract. This paper aims to determine the stresses and strains that can occur in the high density polyethylene pipes when they vertically come under the force of a mechanic squeeze off tool in order to close the natural gas pipelines to conduct under pressure couplings.

\section{Introduction}

The natural gas distribution pipelines at certain times require undergoing certain operations to close the passage of gases in order to achieve the necessary couplings. These couplings can be made under pressure and a related study is required in order to observe the values of the strains and stresses occurring in the process in order to avoid the pipeline giving in when shut. The polyethylene pipes are widely used in natural gas distribution systems and therefore there are extensive studies covering in particular the problems arising from their use. [2]

\section{Forces Occurring due to the Interior Pressure}

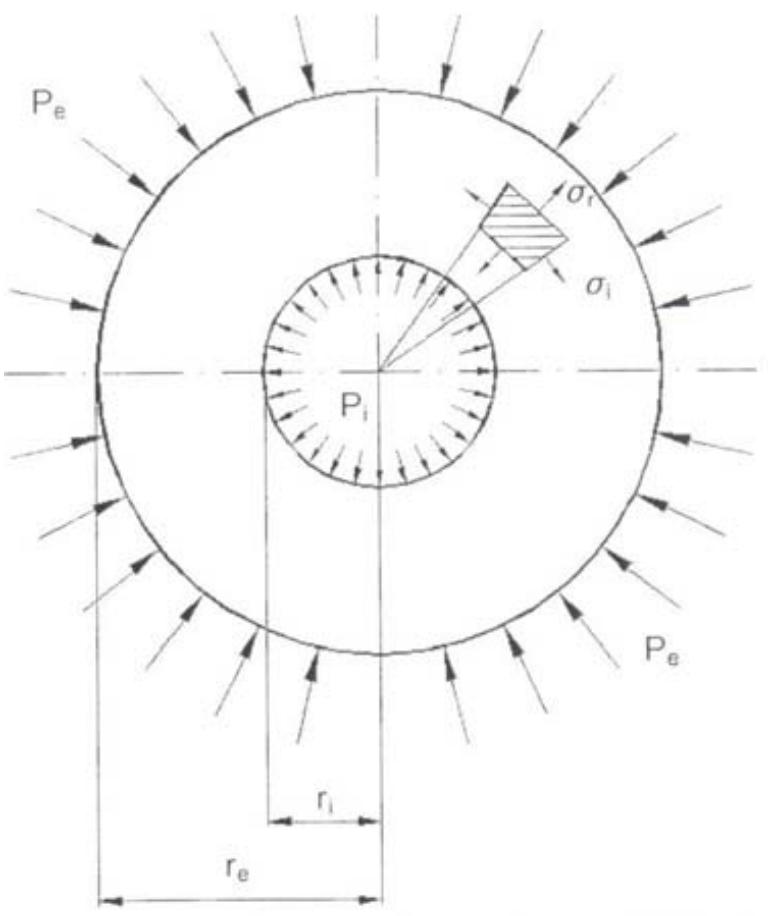

Fig. 1. Distribution of the stresses on the polyethylene pipe according to the application of the external and internal pressures
Under the action of an internal operating pressure $p_{i}$ and of other external pressure $p_{e}$ due to the external factors (permanent weight - earth, temporary weight load, intended pressure with the squeeze off tool) one can observe that stresses occur in all three directions, being able to identify in the pipe cross section both radial, tangential and axial stresses, according to the following relations:

- Axial stress: $\sigma_{a x}=\frac{r_{r} r_{i}^{2}-r_{r} r_{r}^{2}}{r_{\varepsilon}^{2}-r_{i}^{2}}$

- Radial stress:

$\sigma_{\mathrm{r}}=\frac{1}{r_{\mathrm{e}}^{2}-r_{\mathrm{i}}^{2}}\left[\mathrm{p}_{\mathrm{i}} \mathrm{r}_{\mathrm{i}}^{2}-\mathrm{p}_{\mathrm{e}} \mathrm{r}_{\mathrm{e}}^{2}+\left(\mathrm{p}_{\mathrm{e}}-\mathrm{p}_{\mathrm{i}}\right) \frac{r_{\mathrm{r}}^{2} r^{2}}{r^{2}}\right]$

- Tangential stress:

$\sigma_{\mathrm{r}}=\frac{1}{r \frac{n}{\varepsilon}-r_{i}^{2}}\left[\mathrm{p}_{\mathrm{i}} \mathrm{r}_{\mathrm{i}}^{2}-\mathrm{p}_{\mathrm{e}} \mathrm{r}_{\mathrm{e}}^{2}-\left(\mathrm{p}_{\mathrm{e}}-\mathrm{p}_{\mathrm{i}}\right) \frac{r_{\mathrm{e}}^{2} p_{\mathrm{e}}^{2}}{r^{2}}\right]$

The following terms are identified in the three previous relations: $r_{i}-$ internal radius of the polyethylene pipe; $r_{e}-$ external radius of the polyethylene pipe. [2] 


\section{Presentation of the Analysis Method}

The software we used in modeling the numerical simulation under compression on the radial direction of the polyethylene pipes is the Ansys Ls-Dyna software. It was chosen due to its capacity to describe and take into consideration all three types of nonlinearity (material nonlinearity, geometric nonlinearity and boundary conditions nonlinearity). An incremental formulation of the equilibrium equations is used (the revised Lagrangian formulation) based on observing the equilibrium of the deformable body at the time intervals $0, \Delta t, 2 \Delta t, 3 \Delta t, \ldots, n \Delta t$. [1]

The calculation covers all the nodes of the finite elements network from the beginning of the end of its deformation. In the revised Lagrangian formulation, the stiffness matrix is assembled in the geometrical configuration of the current increment, and the results calculated at the end of the increment (stresses, strains and nodal displacements) are associated to the revised nodal geometry according to the calculated displacements. This formulation is the most appropriate among the available ones, for modeling this deformation process, characterized by a permanently changing geometry and by large displacements of certain elements. In connection to this formulation, we defined the material properties associated to the model, which must fully characterize it. [1]

In order to simulate this test of compression on the radial direction, the Ansys software provides the user with a special module, namely Ls-Dyna, by means of which both explicit dynamic analysis and implicit dynamic analysis can be achieved.

\section{Simulation of Pressing the Pipe with the Squeeze Off Tool}

The present analysis is conducted on a PE100 pipe of $90 \mathrm{~mm}$ diameter and $8.2 \mathrm{~mm}$ wall thickness (similar to that experimentally studied in chapter 2 of the present paper), the software used being Lsprepostd for viewing and saving the results. The results were focused on determining the stresses (represented by the von Mises stress and the principal stress on the direction of the force), determining the deformations (represented by the von Mises principal, secondary and equivalent unit strain), the displacement of the pipe in the two radial directions, as well as the variation of the compression force during the deformation process.

Figure 2 shows the assembly upper bar - pipe - lower bar, mashed in finite elements, and figure 3 represents the variation graph of the compression force at the level of the upper bar. The maximum value of this force reached approximately $860[\mathrm{~N}]$.

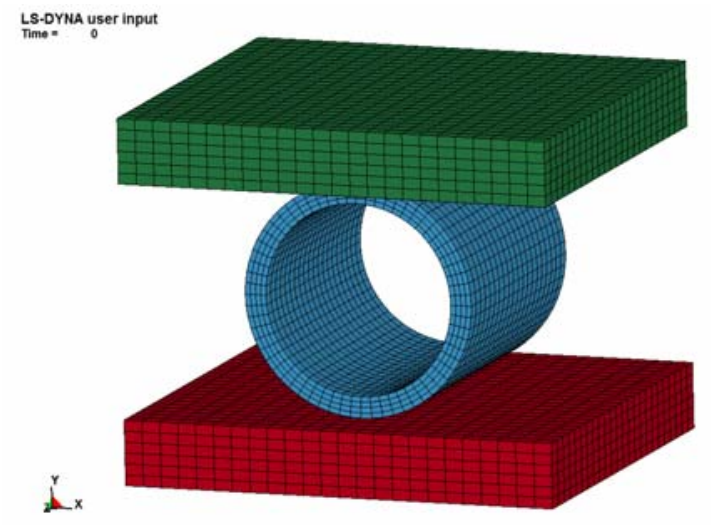

Fig. 2. Assembly upper bar-pipe-lower bar (mashed)

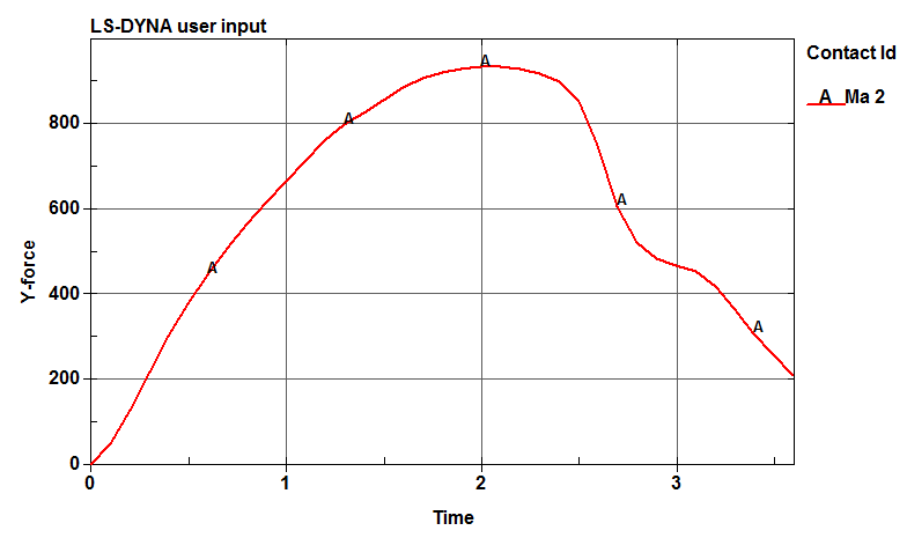

Fig. 3. Variation of the compression force in the upper bar

The value of the pipe material displacement on the radial direction Ox at increment (step of the analysis) 0.3 and respectively 1.3 is lower than that on Oy and that is why we shall present the value registered on Oy. 


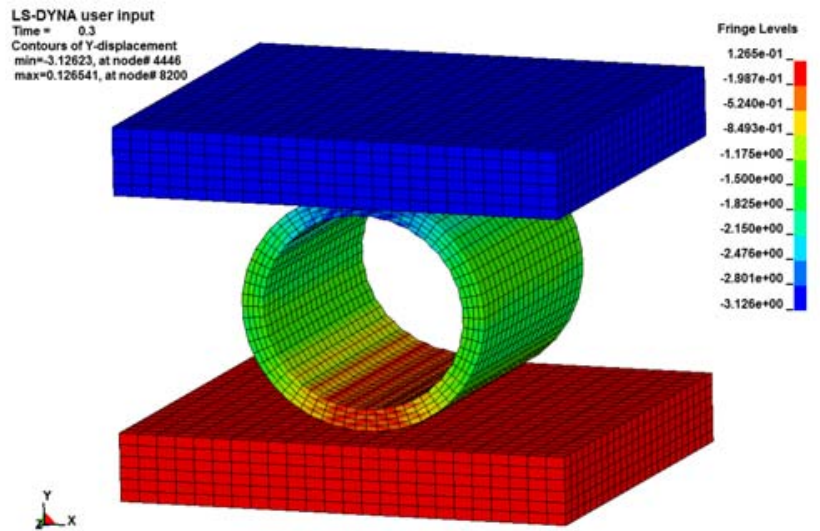

Fig. 4. The displacement of the pipe material on the radial direction $O y$ at increment 0.3

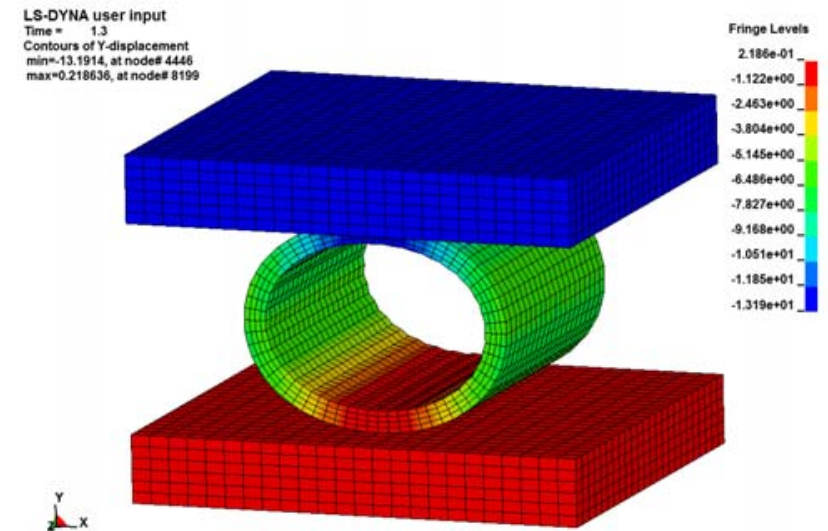

Fig. 5. The displacement of the pipe material on the radial direction $O y$ at increment 1.3

Figures 6 and 7 show the distribution map of the principal strain in the pipe material, at increment (step of the analysis) 0.3 and respectively 1.3 .

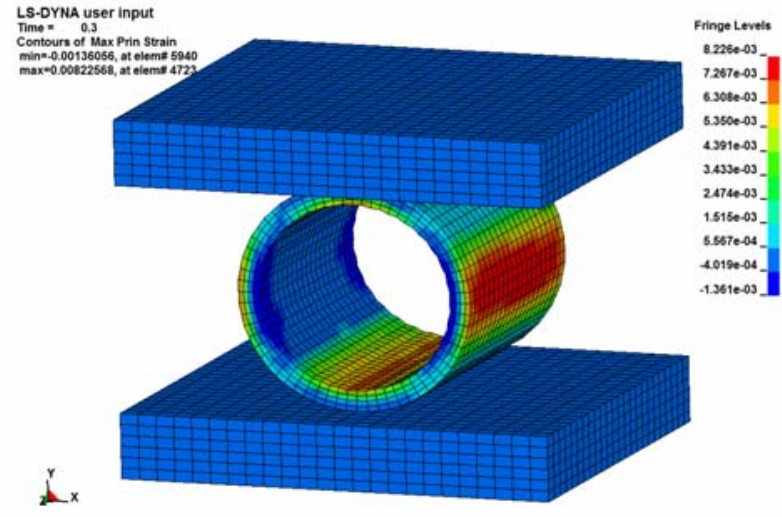

Fig. 6. Map of the principal strain in the pipe material at increment 0.3

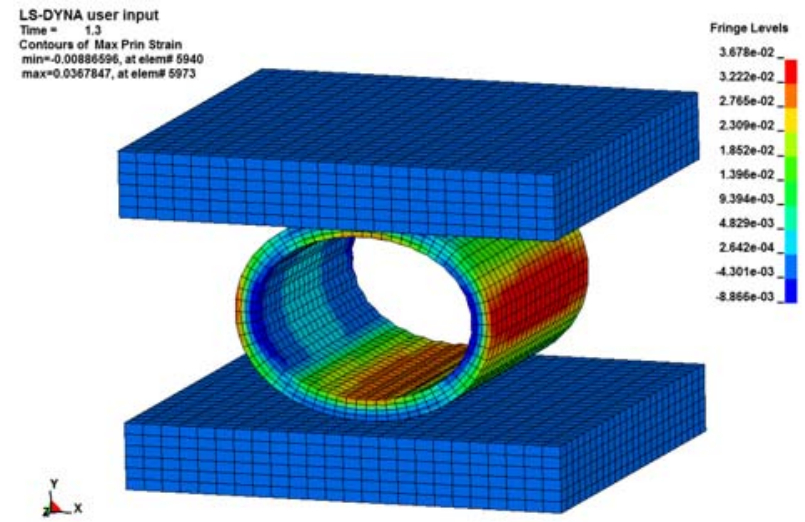

Fig. 7. Map of the principal strain distribution in the pipe material at increment 1.3

Figures 8 and 9 show the map of the distribution of the von Mises strain in the pipe material, at increment (step of the analysis) 0.3 and 1.3.

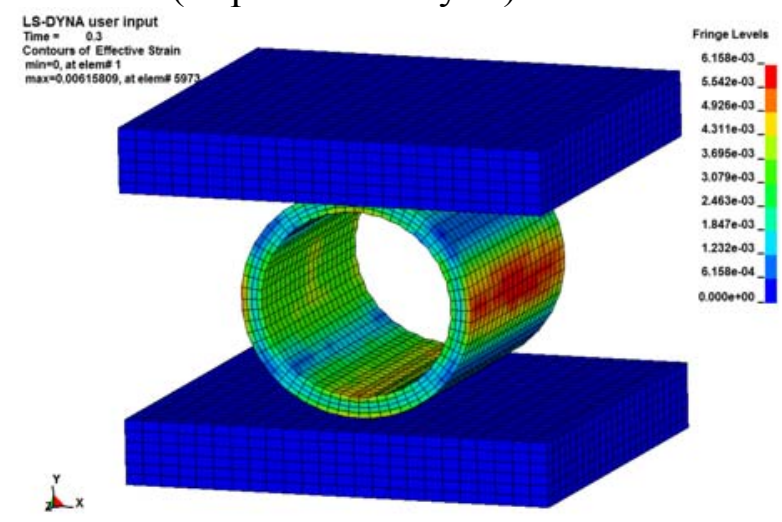

Fig. 8. Map of the von Mises effective strain in the pipe material at increment 0.3

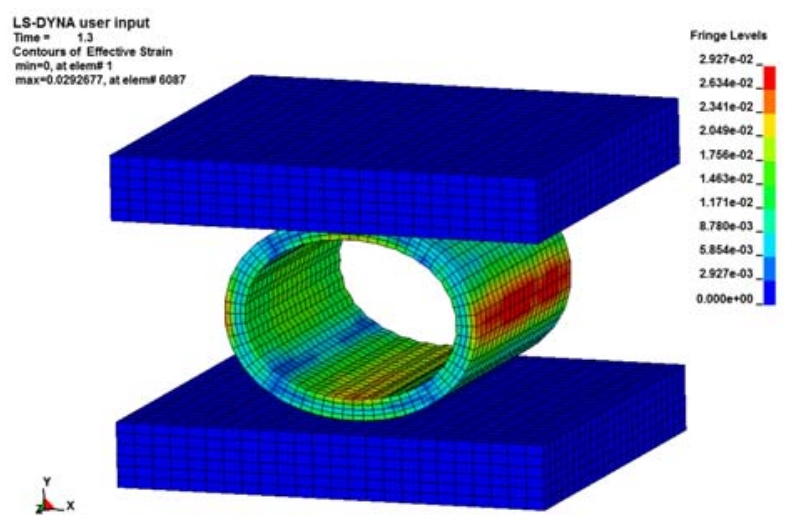

Fig. 9. Map of the von Mises effective strain distribution in the pipe material at increment 1.3

Figures 10 and 11 show the distribution map of the von Mises stresses appearing in the pipe material, at the increment (step of the analysis) 0.3 and 1.3. 


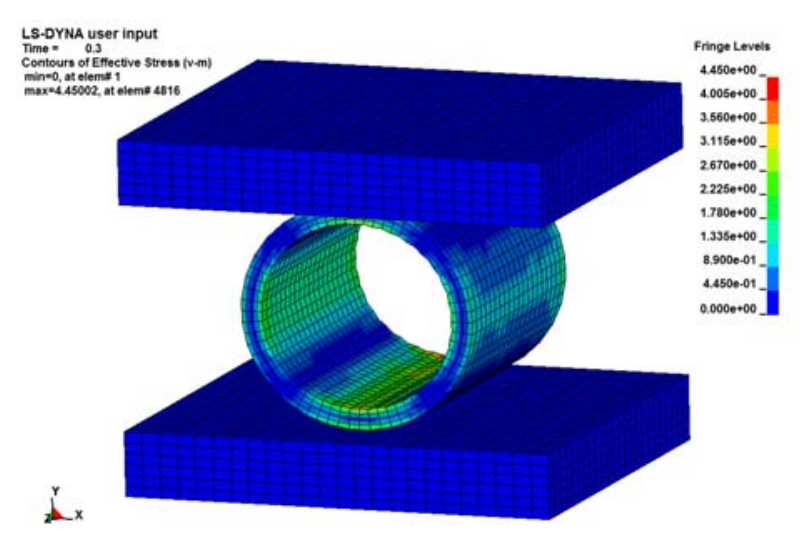

Fig. 10. Map of von Mises stress distribution occurring in the pipe material, at increment 0.3

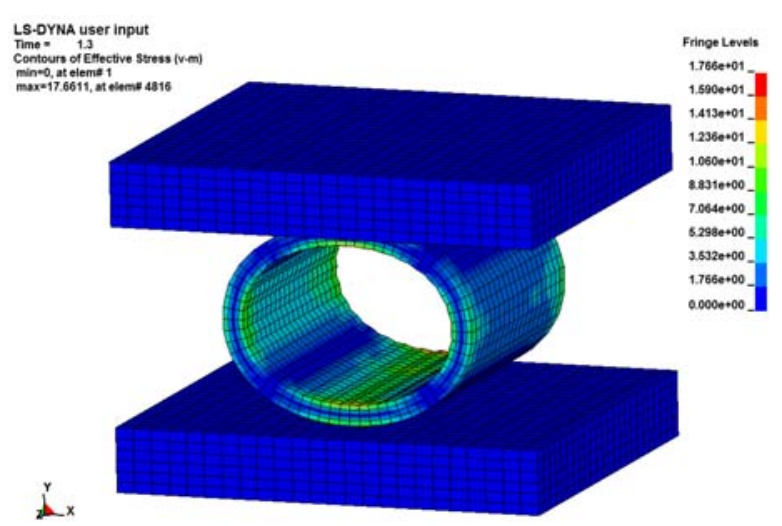

Fig. 11. Map of von Misses stress distribution occurring in the pipe material at increment 1.3

In addition to these types of data presented here, the program allows the determination of many more data, such as: the energy consumption, the amount of mechanical work, the amount of energy consumed by friction, the tensions and strains on various fibers (external, internal, median), etc., which were not presented in the present paper.

These dynamic analyses in the nonlinear field have an increasingly more practical applicability due to the fact that such an analysis can predict the behavior of a polyethylene pipe over time and thus reduce the maintenance costs.

\section{Conclusions}

Following the conducted studies, we observe that a procedure has been established to calculate the stresses occurring in the studied process. The following conclusions were noted related to the performed simulation:

- The presented squeeze off tool should be equipped with a buffer that does not allow the movement of the mobile bar more than the thickness of the two walls of material;

- It must be set on degrees because the wall thickness will change depending on the diameter;

- The simulation was made so that the real work conditions would be consistent with what has been studied;

- The obtained stress is lower than the allowable stress for the high density polyethylene material;

- $\quad$ The strain is also below the maximum value allowed by the literature;

\section{Acknowledgements}

This work was supported by the strategic grant POSDRU/159/1.5/S/133255, Project ID 133255 (2014), co-financed by the European Social Fund within the Sectorial Operational Program Human Resources Development 2007 - 2013.

\section{References}

[1] Avrigean E. Study on Temperature Distribution in the Jointing Fittings for Polyethylene Natural Gas Pipes. 2015 3rd International Conference on Recent Trends in Materials and Mechanical Engineering (ICRTMME 2015), January 15-16, 2015, Auckland, New Zealand.

[2] Balan, M. L. Contributii la utilizarea procedeului de sudare cap la cap a tevilor de polietilena destinate transportului si distributiei gazelor naturale. Doctoral thesis. Sibiu 